\title{
Isolated photon production and pion-photon correlations in high-energy pp and $p A$ collisions
}

\author{
Victor P. Goncalves $\odot,{ }^{1, *}$ Yuri Lima $\odot,{ }^{2}$ Roman Pasechnik, ${ }^{3,4, \dagger}$ and Michal Šumbera ${ }^{4, \$}$ \\ ${ }^{1}$ High and Medium Energy Group, Instituto de Física e Matemática, Universidade Federal de Pelotas, \\ Pelotas, Rio Grande do Sul 96010-900, Brazil \\ ${ }^{2}$ High and Medium Energy Group, Instituto de Física e Matemática, Universidade Federal de Pelotas, \\ Pelotas, RS, 96010-900, Brazil \\ ${ }^{3}$ Department of Astronomy and Theoretical Physics, Lund University, SE-223 62 Lund, Sweden \\ ${ }^{4}$ Nuclear Physics Institute of the CAS, $25068 \check{R} e \check{,}$, Czech Republic
}

(Received 10 March 2020; accepted 7 May 2020; published 18 May 2020)

\begin{abstract}
A phenomenological study of the isolated photon production in high energy $p p$ and $p A$ collisions at RHIC and LHC energies is performed. Using the color dipole approach we investigate the production cross section differential in the transverse momentum of the photon considering three different phenomenological models for the universal dipole cross section. We also present the predictions for the rapidity dependence of the ratio of $p A$ to $p p$ cross sections. As a further test of the formalism, for different energies and photon rapidities we analyze the correlation function in azimuthal angle $\Delta \phi$ between the photon and a forward pion. The characteristic double-peak structure of the correlation function around $\Delta \phi \simeq \pi$ observed previously for Drell-Yan pair production is found for an isolated photon emitted into the forward rapidity region which can be tested by future experiments.
\end{abstract}

DOI: 10.1103/PhysRevD.101.094019

\section{INTRODUCTION}

The isolated (prompt) photon production in $p p$ and $p A$ high-energy collisions represents an attractive and clean probe for strong interactions in soft [1-3] and perturbative regimes of quantum chromodynamics (QCD) [4-6] as well as nuclear effects and medium-induced QCD phenomena [7-9]. This becomes possible due to the absence of QCDinduced final-state interactions associated with absorptive phenomena as well as of an energy loss that is in variance to the di-hadron production channels where the final-state absorptive corrections are typically very large. The prompt photon production in hadron-hadron and hadron-nucleus collisions can be employed to set further constraints on parton density functions (PDFs) in specific kinematic domains not sufficiently well explored by HERA [10-12]. For this purpose, such studies are also in the focus of ongoing and planned measurements at the LHC [2,13-16] and at RHIC [3,17-21].

\footnotetext{
*barros@ufpel.edu.br

†roman.pasechnik@thep.lu.se

*sumbera@ujf.cas.cz
}

Published by the American Physical Society under the terms of the Creative Commons Attribution 4.0 International license. Further distribution of this work must maintain attribution to the author(s) and the published article's title, journal citation, and DOI. Funded by SCOAP.
At very low $x$, for example, the primordial transverse momentum evolution of incoming partons and nonlinear QCD effects such as gluon saturation start to play a significant role whose reliable first-principle analysis represents a long-standing theoretical challenge. In the case of high-energy $p A$ collisions, main issues concern a proper description of initial/final state effects in multiple interactions with a nuclear target. Another widely discussed problem is associated with propagation of partons in the nuclear environment. Such processes, as the Drell-Yan (DY) pair production, studied recently by some of the authors in Refs. [22-24], as well as the isolated photon production at high- $p_{T}$, provide efficient means for phenomenological analysis of various nuclear effects such as the nuclear shadowing and initial-state interactions determined by saturation [25].

In this paper, we investigate the isolated photon production off the proton and nuclear targets in the low- $x$ regime of QCD in the framework of the phenomenological color dipole formalism (see, e.g., Refs. [26-32]). In the dipole picture, the real photon production is considered as $\gamma$ bremsstrahlung off a fast projectile quark propagating through the low- $x$ color field of the target [28] as illustrated in Figs. 1(a) and 1(b). In this case, the photon radiation occurs both after and before the quark scatters off the target and the corresponding amplitudes interfere. As a result of such interference, the photon bremsstrahlung process can be viewed as scattering of a $q \bar{q}$ dipole with a given 


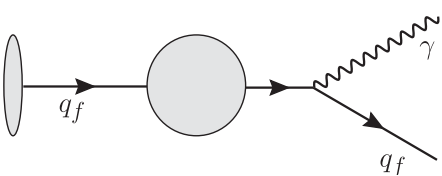

(a)

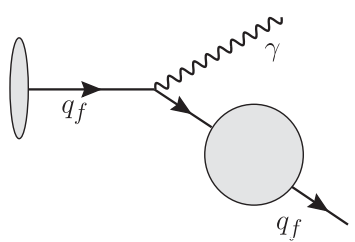

(b)

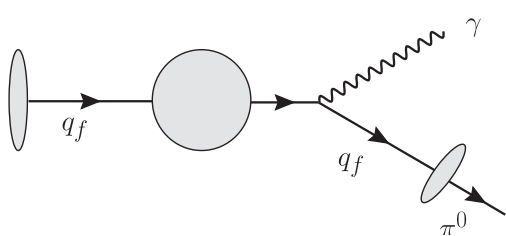

(c)

FIG. 1. (a) and (b) The $\gamma$ bremsstrahlung process off a projectile a quark (antiquark) of flavor $f$ either after or before its interaction with the color field of the target (denoted by a shaded circle), respectively. (c) One of the possible contributions to the pion-photon pair production in the color dipole picture.

transverse separation. This is in variance to the conventional parton model where the same process in the centerof-mass frame is given by the Compton scattering. The difference between both descriptions illustrates the wellknown fact that although cross sections are Lorentz invariant, the partonic interpretation of the corresponding processes depends on the reference frame.

The key ingredients of the dipole formula for the differential cross section of the considered process are the light-cone (LC) wave function of the initial state describing the real photon radiation off the projectile quark as well as the universal dipole-target cross section related to the dipole $S$ matrix, $\sigma_{q \bar{q}}(x, \rho)$, which can be determined phenomenologically, for example, by a fit to the deep inelastic scattering (DIS) data at low- $x$ [33] or to a DrellYan $p p$ data of good quality. At small Bjorken $x$ (or at high energies), the universality of the dipole cross section stems from the fact that color dipoles in QCD are the eigenstates of interaction with a fixed transverse separation, $\rho$ [26].

Remarkably, since the lifetime of partonic fluctuation in the laboratory frame is enhanced by a factor of $\sqrt{s} / m_{p}$ with respect to the lifetime in the center-of-mass system, the phenomenological dipole approach appears to effectively take into account the higher-order QCD corrections. For example, it provides the predictions for the DY process at the same level of precision as the next-to-leading-order (NLO) collinear factorization framework [31]. Besides, as a consequence of universality, the dipole formulation provides a unified description of a variety of inclusive and diffractive observables of particle production processes in lepton-hadron, hadron-hadron, hadron-nucleus, and nucleus-nucleus collisions at high energies (for particular examples, see, e.g., Refs. [27-29,32,34-36]). In the highenergy limit, the projectile quark effectively probes a dense gluonic field in the target with the dipole cross section effectively accounting for the nonlinear effects due to multiple scatterings.

The goal of the current work is the following. First, we update the previous studies presenting predictions for the transverse momentum distribution of isolated photons produced at the RHIC and LHC energies. Moreover, we also make predictions for the ratio between the proton-lead $(p P b)$ and proton-proton $(p p)$ cross sections at the LHC for different values of the photon (pseudo)rapidity. Second, we present a detailed analysis of the azimuthal correlation between the photon and a pion that emerges from a projectile quark hadronization at forward rapidities ${ }^{1}$ [see Fig. 1(c)]. In this paper, we present new results for such an observable for $p p$ collisions at RHIC $(\sqrt{s}=500 \mathrm{GeV})$ and LHC $(\sqrt{s}=14 \mathrm{TeV})$, as well for $p A u$ collisions at RHIC $(\sqrt{s}=200 \mathrm{GeV})$ and $p P b$ collisions at the LHC $(\sqrt{s}=8.8 \mathrm{TeV})$. To estimate the related theoretical uncertainties in our predictions, we consider three different approaches to saturation effects $[33,42,43]$.

The paper is organized as follows. In Sec. II, a brief overview of isolated photon production in the color dipole framework is provided. In Sec. III, we present our numerical results for the transverse momentum distributions of the produced isolated photon as well as the $p A$-to- $p p$ ratio of the production cross sections. Furthermore, the pionphoton azimuthal correlation function is evaluated for $p p$ and $p A$ collisions at the characteristic RHIC and LHC energies for different photon and pion rapidities. Finally, in Sec. IV, our main conclusions are summarized.

\section{COLOR DIPOLE PICTURE OF REAL PHOTON BREMSSTRAHLUNG}

Consider first the isolated photon production in $p p$ collisions in the target rest frame. In the high energy limit, Figs. 1(a) and 1(b) in the impact-parameter space can be represented as a convolution of the LC wave function of the projectile quark $|q\rangle$ fluctuation into its lowest $|q \gamma\rangle$ Fock state and a scattering amplitude of a quark off the target $T$ at a given impact parameter [27,28]. Here, $T$ denotes either the proton $p$ or a nucleus target with an atomic mass $A$.

In what follows, we work in terms of usual LC (longitudinal) momentum fractions of the isolated photon, $x_{1}$ and $x_{2}$, taken from the incoming proton momenta $p_{1}$ and $p_{2}$, respectively, such that

$x_{1}=\frac{p_{\gamma}^{+}}{p_{1}^{+}}=\frac{p_{T}}{\sqrt{s}} e^{\eta}, \quad x_{2}=\frac{p_{\gamma}^{-}}{p_{2}^{-}}=\frac{p_{T}}{\sqrt{s}} e^{-\eta}, \quad x_{1}-x_{2} \equiv x_{F}$,

\footnotetext{
${ }^{1}$ Similar correlations in di-hadron, real photon-hadron, and dilepton-hadron channels have previously been reported in Refs. [37-41].
} 
where $p_{T}, \eta$, and $x_{F}$ are the transverse momentum, pseudorapidity, and the Feynman variable of the photon. The initial-state quark $|q\rangle$ and the final-state quark accompanied by a Weizäcker-Williams photon, $|q \gamma\rangle$, propagate at different impact parameters. Indeed, because of the $\gamma$ bremsstrahlung the final quark gets a transverse shift with respect to the initial one, $\Delta \mathbf{r}=\alpha \boldsymbol{\rho}$, where $\alpha$ is the fractional LC momentum taken by the radiated photon off the projectile quark and $\boldsymbol{\rho}$ is the quark- $\gamma$ transverse separation.

The amplitudes in Figs. 1(a) and 1(b) corresponding to the scattering of $|q\rangle$ and $|q \gamma\rangle$ Fock states off the target $T$, respectively, interfere. As a result, the matrix element squared for the isolated photon production integrated over the impact parameter of the initial quark is expressed in terms of the universal $q \bar{q}$ dipole-target cross section $\sigma_{q \bar{q}}^{T}(\Delta \mathbf{r}, x)$ as a function of the transverse separation $\Delta \mathbf{r}$ and the standard Bjorken variable of the process $x$, which is taken to be equal to $x_{2}$ in what follows. The cross section for the real photon production differential in photon transverse momentum $p_{T}$ and pseudorapidity $\eta$,

$$
\frac{d \sigma(p T \rightarrow \gamma X)}{\mathrm{d}^{2} p_{T} d \eta}=\frac{2 p_{T}}{\sqrt{s}} \cosh (\eta) \frac{x_{1}}{x_{1}+x_{2}} \sum_{f} \int_{x_{1}}^{1} \frac{d \alpha}{\alpha^{2}}\left[q_{f}\left(x_{1} / \alpha, \mu_{F}^{2}\right)+\bar{q}_{f}\left(x_{1} / \alpha, \mu_{F}^{2}\right)\right] \frac{d \sigma^{f}(q T \rightarrow q \gamma X)}{d \ln \alpha d^{2} p_{T}}
$$

is typically found in terms of the unpolarized projectile quark (antiquark) collinear PDFs $q_{f}\left(\bar{q}_{f}\right)$ corresponding to (valence and sea) flavor $f=u, d, s, c$ as functions of the momentum fraction of the projectile quark taken from the parent nucleon $x_{q}=x_{1} / \alpha$ and the QCD factorization scale $\mu_{F}=p_{T} \equiv\left|\mathbf{p}_{T}\right|$. The differential cross section of the high- $p_{T}$ real photon production in the quark-target scattering subprocess is represented in the dipole picture as

$$
\begin{aligned}
\frac{d \sigma^{f}(q T \rightarrow q \gamma X)}{d \ln \alpha d^{2} p_{T}}= & \frac{1}{(2 \pi)^{2}} \int d^{2} \rho_{1} \mathrm{~d}^{2} \rho_{2} \exp \left[i \mathbf{p}_{T} \cdot\left(\boldsymbol{\rho}_{1}-\boldsymbol{\rho}_{2}\right)\right] \Psi\left(\alpha, \boldsymbol{\rho}_{1}, m_{f}\right) \Psi^{*}\left(\alpha, \boldsymbol{\rho}_{2}, m_{f}\right) \\
& \times \frac{1}{2}\left[\sigma_{q \bar{q}}^{T}\left(\alpha \boldsymbol{\rho}_{1}, x_{2}\right)+\sigma_{q \bar{q}}^{T}\left(\alpha \boldsymbol{\rho}_{2}, x_{2}\right)-\sigma_{q \bar{q}}^{T}\left(\alpha\left|\boldsymbol{\rho}_{1}-\boldsymbol{\rho}_{2}\right|, x_{2}\right)\right]
\end{aligned}
$$

where $m_{f}$ is the constituent quark mass and $\Psi\left(\alpha, \rho, m_{f}\right)$ is the $\mathrm{LC}$ wave function of the real photon radiation off a quark with flavor $f$. Following Ref. [33], we take the constituent quark mass values to be $m_{u}=m_{d}=m_{s}=0.14 \mathrm{GeV}$ and $m_{c}=$ $1.4 \mathrm{GeV}$ in our numerical analysis below. For the cross section differential in photon $\mathbf{p}_{T}$ the quark- $\gamma$ transverse separations amplitude and its conjugate are considered to be different and are denoted as $\boldsymbol{\rho}_{1,2}$. In this case, the overlap of the photon bremsstrahlung wave functions in Eq. (3), summed over the transverse polarizations of the radiated hard photon, reads

$$
\Psi\left(\alpha, \boldsymbol{\rho}_{1}, m_{f}\right) \Psi^{*}\left(\alpha, \boldsymbol{\rho}_{2}, m_{f}\right)=\frac{\alpha_{\mathrm{em}} e_{f}^{2}}{2 \pi^{2}}\left\{m_{f}^{2} \alpha^{4} \mathrm{~K}_{0}\left(\tau \rho_{1}\right) \mathrm{K}_{0}\left(\tau \rho_{2}\right)+\left[1+(1-\alpha)^{2}\right] \tau^{2} \frac{\boldsymbol{\rho}_{1} \cdot \boldsymbol{\rho}_{2}}{\rho_{1} \rho_{2}} \mathrm{~K}_{1}\left(\tau \rho_{1}\right) \mathrm{K}_{1}\left(\tau \rho_{2}\right)\right\},
$$

where $\bar{\alpha} \equiv 1-\alpha, \alpha_{\mathrm{em}}$ is the fine structure constant, $e_{f}$ is the charge of the projectile quark, $\rho_{1,2} \equiv\left|\rho_{1,2}\right|, \tau=\alpha m_{f}$, and the modified Bessel functions of the second kind are denoted as $\mathrm{K}_{0,1}$. In fact, the photon transverse momentum provides a hard scale for the considering process that ensures the validity of the perturbative approximation which has been used in the computation of the photon wave function in Eq. (4).

We would like to analyze the correlation in the azimuthal angle between the final-state photon and a hadron emerging due to hadronization of the projectile (anti)quark associated with the photon radiation. An analogous analysis for the DY process with a deeply virtual photon has been performed earlier in the impact parameter representation in Ref. [30], although the corresponding numerical analysis is very challenging. More recently, in Ref. [24] a numerical calculation of the differential DY cross section derived in Refs. [41,44-46] has been performed directly in momentum representation. We adopt the same formalism for the considering case of real high- $p_{T}$ photon production in association with the leading hadron $h$, namely,

$$
\frac{d \sigma(p T \rightarrow h \gamma X)}{d \eta d y_{h} d^{2} p_{T} d^{2} p_{T}^{h}}=\frac{\alpha_{\mathrm{em}}}{2 \pi^{2}} \int_{\frac{x_{h}}{1-x_{1}}}^{1} \frac{\mathrm{d} z_{h}}{z_{h}^{2}} \sum_{f} e_{f}^{2} D_{h / f}\left(z_{h}, \mu_{F}^{2}\right) x_{p} q_{f}\left(x_{p}, \mu_{F}\right) S_{\perp} F_{T}\left(x_{g}, k_{T}^{g}\right) \frac{\bar{z} z^{2}\left(1+\bar{z}^{2}\right) k_{T}^{g} 2}{P_{T}^{2}\left(\mathbf{P}_{T}+z \mathbf{k}_{T}^{g}\right)^{2}},
$$


where the key kinematical variables are determined as follows:

$$
x_{h} \simeq \frac{p_{T}^{h}}{\sqrt{s}} e^{y_{h}}, \quad x_{p}=x_{1}+\frac{x_{h}}{z_{h}}, \quad z=\frac{x_{1}}{x_{p}}, \quad x_{g}=x_{1} e^{-2 \eta}+\frac{x_{h}}{z_{h}} e^{-2 y_{h}},
$$

$$
\mathbf{k}_{T}^{g}=\mathbf{p}_{T}+\mathbf{k}_{T}^{q}, \quad \mathbf{P}_{T}=\bar{z} \mathbf{p}_{T}-z \mathbf{k}_{T}^{q}, \quad \mathbf{k}_{T}^{q}=\frac{\mathbf{p}_{T}^{h}}{z_{h}} .
$$

Here, for simplicity, we are considering the light quark flavors $f=u, d, s$ only and neglect terms proportional to $m_{f}$ due to $p_{T} \gg m_{f}$. In Eq. (5), $D_{h / f}$ stands for the fragmentation function of the projectile quark $q_{f}$ (which has emitted the photon) into a final-state (light) hadron $h$ carrying the transverse momentum $\mathbf{p}_{T}^{h}$ that is supposed to be detected in a measurement. The remaining kinematic variables are defined as follows: $y_{h}$ is the rapidity of the hadron $h$ in the final state, respectively, $z_{h}$ and $x_{h}$ are the LC momentum fractions taken by the hadron $h$ from the parent quark $q_{f}$ and the incoming proton, $\mathbf{P}_{T}$ is the relative transverse momentum between the photon and the quark $q_{f}, \mathbf{k}_{T}^{q}$ is the transverse momentum of the projectile quark $q$ (before it fragments into a hadron $h$ ), and $\mathbf{k}_{T}^{g}$ is the transverse momentum of the exchanged gluon in the $t$ channel. Finally, $S_{\perp}$ denotes the transverse area of the considered target $T$ whose explicit form is irrelevant for our purposes here, $F_{T}\left(x_{g}, k_{T}^{g}\right)$ represents the so-called unintegrated gluon distribution function (UGDF) in the target $T$. In the saturation regime and for the soft gluon $k_{T}^{g}$, the latter can be found in terms of a Fourier transform of the dipole cross section $\sigma_{q \bar{q}}^{T}$ [39]. Note the momentum fractions $z$ and $x_{p}$ share the same physical meaning as $\alpha$ and $x_{q}$ introduced above in Eq. (2), respectively. A different notation is used here since $z$ and $x_{p}$ are now related to the hadron kinematic variables $z_{h}, y_{h}$, and $p_{T}^{h}$ in the final state.

One of the important observables sensitive to the dynamics of saturation is the correlation function $C(\Delta \phi)$ in azimuthal angle $\Delta \phi$ between the final state photon and hadron (for more details, see, e.g., Ref. [24]). Assuming the isolated photon to be a trigger particle, the correlation function can be built as follows:

$C(\Delta \phi)=\frac{2 \pi \int_{p_{T}, p_{T}^{h}>p_{T}^{\text {cut }}} d p_{T} p_{T} d p_{T}^{h} p_{T}^{h} \frac{d \sigma(p T \rightarrow h \gamma X)}{d \eta d y_{h} d^{2} p_{T} d^{2} p_{T}^{h}}}{\int_{p_{T}>p_{T}^{\text {cut }}} d p_{T} p_{T} \frac{d \sigma(p T \rightarrow \gamma X)}{d \eta d^{2} p_{T}}}$,

in terms of the low cutoff $p_{T}^{\text {cut }}$ on transverse momenta of the resolved $\gamma$ and $h$. In the denominator, we have the cross section for inclusive photon production. For consistency, the latter can be straightforwardly obtained by integrating photon-hadron cross section in Eq. (5) over the hadron momentum and rapidity as well as over $\Delta \phi$. This way, one arrives at the following expression:

$$
\frac{d \sigma(p T \rightarrow \gamma X)}{d \eta d^{2} p_{T}}=\frac{\alpha_{\mathrm{em}}}{2 \pi^{2}} \int_{x_{1}}^{1} \frac{d z}{z} \int d^{2} k_{T}^{g} \sum_{f} e_{f}^{2} x_{p} q_{f}\left(x_{p}, \mu_{F}\right) S_{\perp} F_{T}\left(x_{g}, k_{T}^{g}\right) \frac{z^{2}\left(1+\bar{z}^{2}\right) k_{T}^{g 2}}{p_{T}^{2}\left(\mathbf{p}_{T}-z \mathbf{k}_{T}^{g}\right)^{2}} .
$$

For the numerical analysis of the isolated photon observables we need to specify a reliable parametrization for the dipole cross section [26], $\sigma_{q \bar{q}}^{T}(r, x)$. The latter contains important information about possible nonlinear QCD (or saturation) effects in the hadronic state (see for a detailed discussion of saturation phenomena, e.g., Ref. [42]). In the case of $p p$ collisions, we should specify the universal dipole cross section off the proton target. Because of the universality of dipoles as eigenstates of interaction in QCD, such a quantity is typically obtained from a phenomenological analysis of the precision data on DIS available from the HERA collider. For comparison with previous results existing in the literature, we traditionally consider the phenomenologically very successful Golec-Biernat-Wusthoff (GBW) model [33] relying on a simple saturated ansatz

$$
\sigma_{q \bar{q}}^{p}(r, x)=\sigma_{0}\left(1-e^{-\frac{r^{2} Q_{s, p}^{2}(x)}{4}}\right)
$$

with the proton saturation scale

$$
Q_{s, p}^{2}(x)=Q_{0}^{2}\left(\frac{x_{0}}{x}\right)^{\lambda}
$$

where the model parameters $Q_{0}^{2}=1 \mathrm{GeV}^{2}, x_{0}=3.04 \times 10^{-4}$, $\lambda=0.288$, and $\sigma_{0}=23.03 \mathrm{mb}$ were obtained from the fit to the DIS data. Besides, we consider the solution of the Balitsky-Kovchegov equation $[47,48]$ with running coupling obtained in Ref. [43] as an alternative model for the dipole-proton cross section, denoted as AAMQS hereafter. Likewise, its initial conditions were constrained by a fit to the HERA DIS data. Finally, yet another phenomenological saturation model for $\sigma_{q \bar{q}}^{p}(r, x)$ based upon the color glass condensate (CGC) approach [49]

$\sigma_{q \bar{q}}^{p}(r, x)=\sigma_{0} \times \begin{cases}\mathcal{N}_{0}\left(\frac{r Q_{s, p}}{2}\right)^{2\left(\gamma_{s}+\frac{\ln \left(2 / r Q_{s, p}\right)}{\kappa \lambda Y}\right)} & r Q_{s, p} \leq 2 \\ 1-\exp ^{-A \ln ^{2}\left(B r Q_{s, p}\right)} & r Q_{s, p}>2\end{cases}$

has been utilized for comparison as well as to estimate the sensitivity of our predictions to dynamics of the saturation 
effects. Here, $\kappa=\chi^{\prime \prime}\left(\gamma_{s}\right) / \chi^{\prime}\left(\gamma_{s}\right)$, where $\chi$ is the LO Balitsky-Fadin-Kuraev-Lipatov characteristic function, and the coefficients $A$ and $B$ are uniquely determined from the continuity condition for the dipole cross section and its derivative with respect to $r Q_{s, p}$ at $r Q_{s, p}=2$.

While the dipole cross section off the proton target is well-constrained and tested by ample $e p$ and $p p$ phenomenology, in the case of a heavy nucleus target the data are not as precise as for the proton one while the modeling of the corresponding dipole cross section is still a subject of continuous debates. One possible alternative present in several studies in the literature is to consider the GlauberMueller (GM) approach [50,51] based upon resummation of all the multiple elastic rescattering diagrams for the $q \bar{q}$ dipole propagation through the nucleus target. In this model, the dipole-nucleus cross section reads

$\sigma_{q \bar{q}}^{A}(r, x)=2 \int d^{2} b_{A}\left\{1-\exp \left[-\frac{1}{2} \sigma_{q \bar{q}}^{p}(r, x) T_{A}\left(b_{A}\right)\right]\right\}$,

where $T_{A}\left(b_{A}\right)$ is the nuclear thickness function that is typically obtained from the Woods-Saxon distribution for the nuclear density normalized to the atomic mass $A$, and $b_{A}$ is the impact parameter of the dipole with respect to the nucleus center. Another possibility is to consider a solution to the running-coupling Balitsky-Kovchegov (rcBK) equation for the nuclear case discussed, e.g., in Refs. [52,53], which takes into account mutual interactions of the gluonic ladders exchanged between the dipole and the nucleus. These two approaches include different diagrams and have distinct predictions for the onset of the saturation phenomena.

In the next section, we perform a numerical analysis of the nuclear modification factor $R_{p A}$ (the $p A$-to- $p p$ ratio of the differential cross sections) and the azimuthal correlation for isolated photon production and compare predictions obtained with these two models for the dipole cross section off the nucleus.

\section{NUMERICAL RESULTS}

In this section, we present numerical results for the isolated photon production in the $p p \rightarrow \gamma X$ process in the framework of color dipole formalism. In this analysis, we employ three phenomenological parametrizations for the dipole cross section discussed above and use the CT10 NLO parametrization for the projectile quark PDFs [54] (both sea and valences quarks are included).

In Fig. 2 we compare our predictions with the PHENIX data [55] for isolated photon production at midrapidity in $p p$ collisions at $\sqrt{s}=0.2 \mathrm{TeV}$ obtained by using three distinct models for the dipole cross section off the proton target. We can see that the GBW and AAMQS models describe the data quite well while the CGC model underestimates the data. Note that our results rely on existing parametrizations for the dipole cross section fitted to the HERA data, without any additional free parameters. In particular, no NLO $K$ factor has been imposed in the calculations, in contrast to the collinear QCD approach where such a factor is required.

In Fig. 3 we present our predictions for isolated photon production in $p p$ collisions at $\sqrt{s}=0.5 \mathrm{TeV}$ and for two distinct values for the photon pseudorapidity, $\eta=2$ (left panel) and $\eta=4$ (right panel). Here, we have selected forward rapidities in order to probe small values of $x_{2}$ in the validity domain of the dipole approach. We expect that in this case the direct photon $p_{T}$ spectra are more sensitive to the treatment of the saturation effects.

The results presented in Fig. 3 (left panel) confirm that this expectation is valid already for $\eta=2$. Here, the predictions for the photon spectrum are similar at small $p_{T}$ 's but start to deviate significantly at $p_{T}>6 \mathrm{GeV}$. In particular, the AAMQS result, associated with the solution of the rcBK equation, predicts larger values for the spectra at large $p_{T}$ 's than those for the GBW and CGC models.

In contrast, the results for $\eta=4$ shown in Fig. 3 (right panel) indicate that at such large rapidities one cannot distinguish the predictions of the different dipole models. Indeed, the dipole approach becomes more precise for smaller values of $x_{2}$. In addition, such small difference between the dipole model predictions is partly due to the fact that here we probing the photon $p_{T}$ spectrum in the edge of the phase space where its behavior is determined essentially by the kinematics of the process.

Our predictions for $p p$ collisions at the LHC energy $(\sqrt{s}=14 \mathrm{TeV})$ and for two different values of the photon pseudorapidity are presented in Fig. 4. Similar to what was observed at RHIC energies, we found that the AAMQS

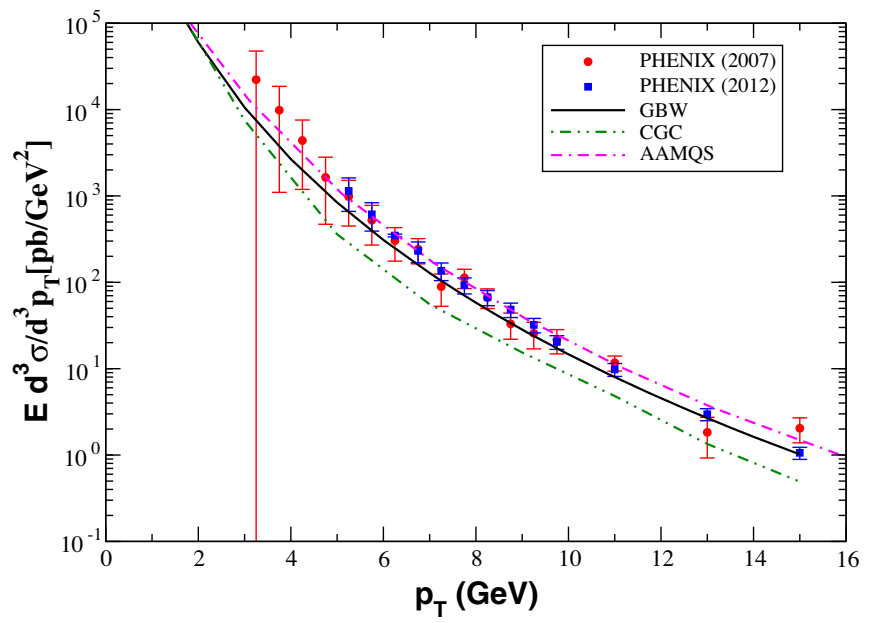

FIG. 2. The isolated photon transverse-momentum spectra in $p p$ collisions at $\sqrt{s}=0.2 \mathrm{TeV}$ and at midrapidity, $\eta=0$, obtained using the different models for the dipole cross section discussed in the text. The experimental data are from the PHENIX experiment [55]. 

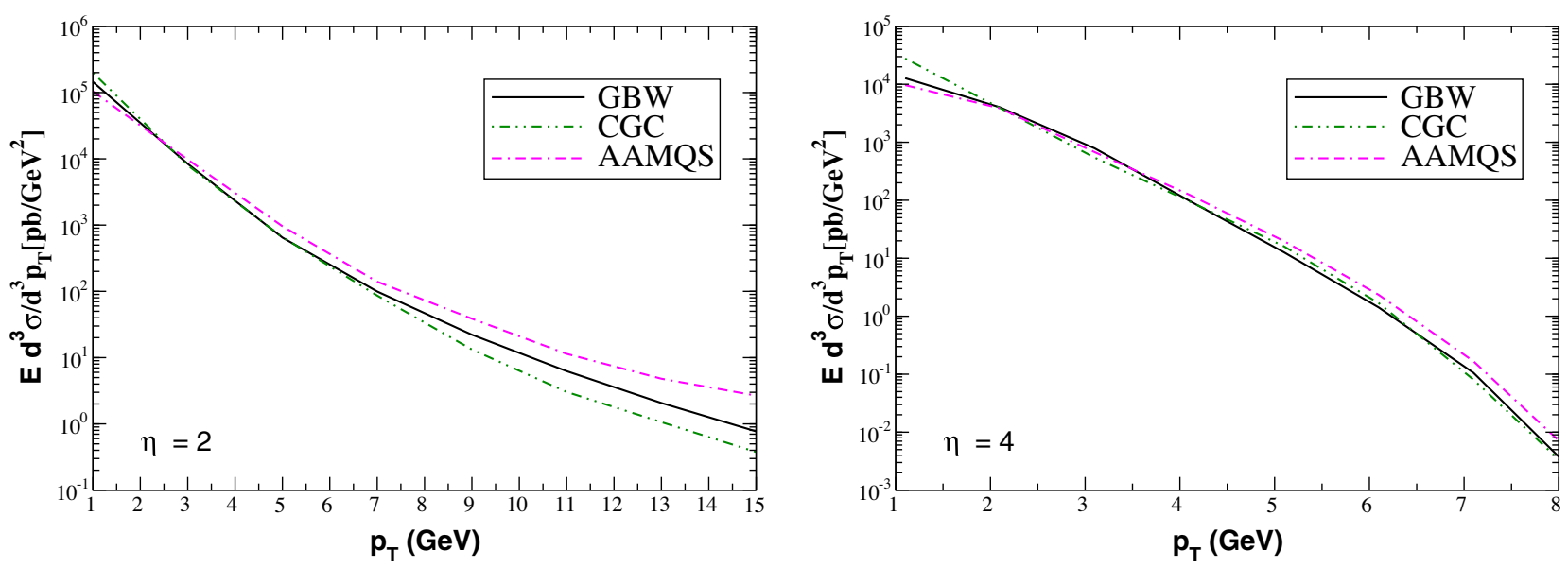

FIG. 3. The isolated photon transverse-momentum spectra in $p p$ collisions at $\sqrt{s}=0.5 \mathrm{TeV}$ of the RHIC experiments for two distinct values of the photon pseudorapidity $\eta$. The results are presented for different models for the dipole cross section discussed in the text.
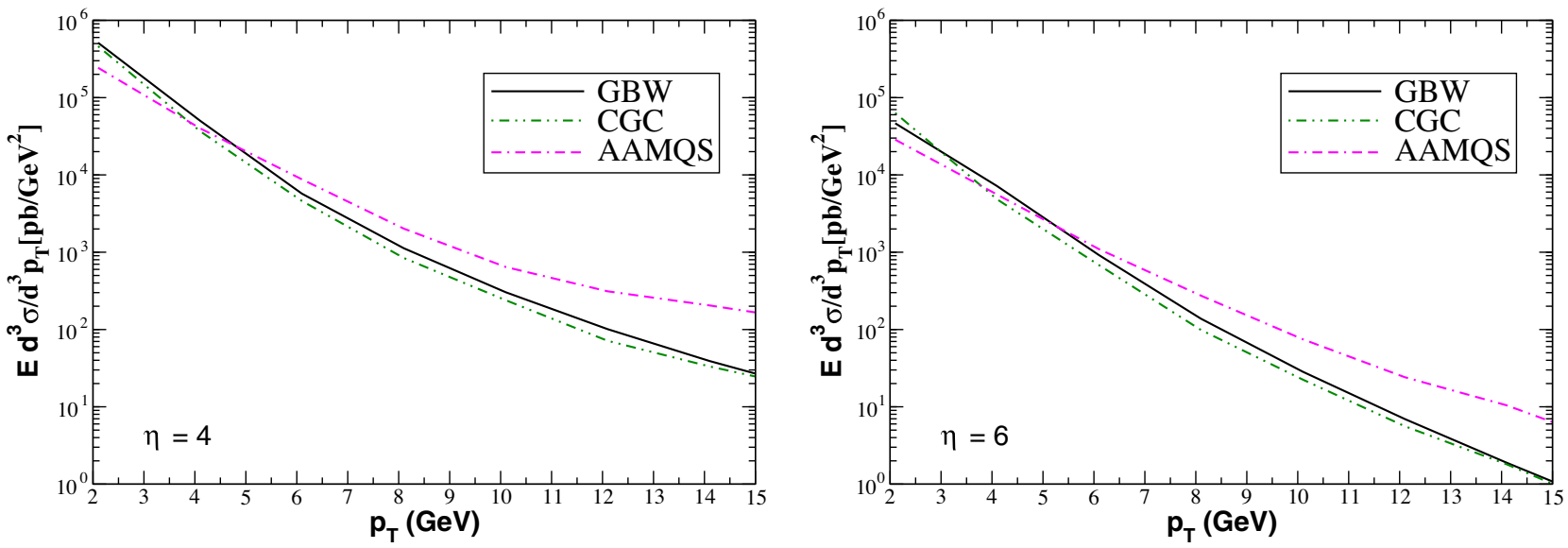

FIG. 4. The isolated photon transverse-momentum spectra in $p p$ collisions at $\sqrt{s}=14 \mathrm{TeV}$ of the LHC experiments for two distinct values of the pseudorapidity $\eta$. The results are presented for different models for the dipole cross section discussed in the text.

prediction yields a higher spectrum than the other models, particularly, at large photon transverse momenta while the CGC and GBW parametrizations provide similar predictions. In principle, future experimental data at large $p_{T}$ can be used to discriminate between the AAMQS and GBW models. Note that at small $p_{T}$, however, the AAMQS prediction becomes slightly below the GBW one.

To estimate the impact of the nuclear effects in the predictions for the isolated photon production in protonlead $(p P b)$ collisions at the LHC $(\sqrt{s}=14 \mathrm{TeV})$, in Fig. 5 we present our predictions for the photon transverse momentum dependence of the nuclear modification factor $R_{p A}$ defined as a ratio between the nuclear and proton differential cross sections, normalized by the atomic mass $A$. The predictions derived using the GlauberMueller approach for the dipole-nucleus cross section, Eq. (13), are denoted as "GM" in the figure. This model predicts that $R_{p A}$ becomes smaller than one at small $p_{T}$ while the nuclear effects become essentially negligible at large $p_{T}$. Moreover, the position of the maximum depends on the rapidity and shifts toward larger $p_{T}$ 's when the rapidity is increased. In contrast, when a solution of the BK equation with QCD running coupling (denoted as "rcBK" in the figure) is used to evaluate the photon spectra in $p p$ and $p P b$ collisions at forward rapidities, the ratio $R_{p A}$ is below unity in the whole considered range of $p_{T}$ 's, in agreement with the results obtained in Ref. [8]. Our results indicate that a future experimental analysis of the nuclear modification factor at forward rapidities can be very useful to discriminate between these two approaches.

To probe the underlying dynamics of particle production at forward rapidities, one should study other observables sensitive to QCD dynamics at small $x$, in particular to QCD nonlinear and saturation phenomena. An appealing possibility is to consider the correlation function $C(\Delta \phi)$ defined in Eq. (8), which is strongly sensitive to the details of the dipole model. The previous results for the associated DY + pion production $[22,24]$ have demonstrated that the 


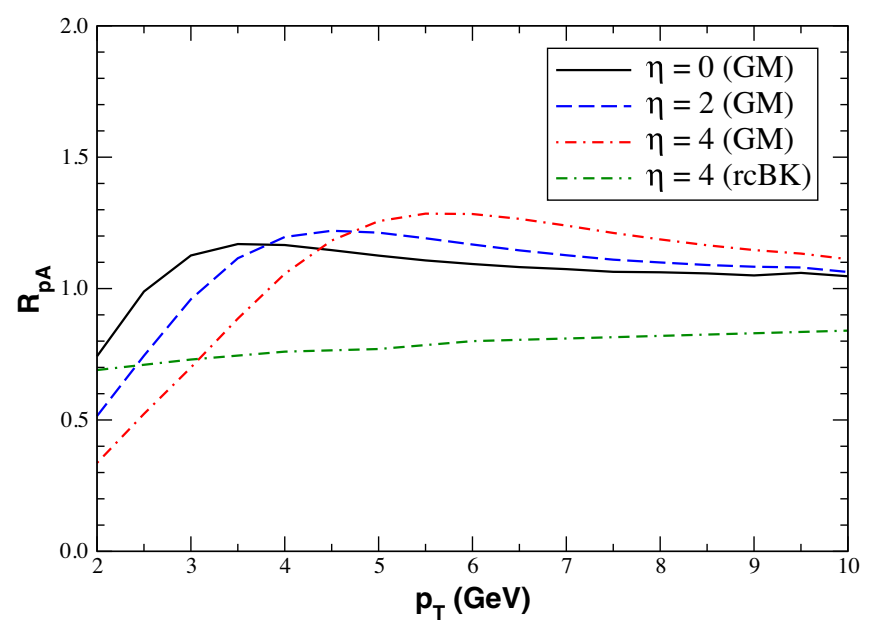

FIG. 5. Transverse momentum dependence of the normalized nuclear modification factor $R_{p A}$ for isolated photon production in proton-lead collisions $(A=208)$ at the $\operatorname{LHC}(\sqrt{s}=8.8 \mathrm{TeV})$ for several selected values of the photon pseudorapidity $\eta$ and for two distinct (GM and rcBK) models of the dipole-nucleus cross section.

effect of saturation implies a notable smearing of the backto-back scattering profile predicted by the standard collinear formalism. An addition of the NLO corrections in the collinear framework would not account for a dip found at $\Delta \phi=\pi$ in the correlation function which is a direct manifestation of the saturation phenomenon.

Our goal here is to make the corresponding predictions for the isolated photon + pion $h=\pi$ associated production in $p p$ and $p A$ collisions at RHIC and LHC energies. As was typically done in earlier studies, let us initially consider the GBW model for the dipole cross section off the proton target which corresponds to the soft UGDF in the proton

$$
F_{p}\left(x_{g}, k_{T}^{g}\right)=\frac{1}{\pi Q_{s, p}^{2}\left(x_{g}\right)} e^{-k_{T}^{g} / Q_{s, p}^{2}\left(x_{g}\right)},
$$

with the saturation scale given in Eq. (11). Following Ref. [22], the nuclear UGDF, $F_{A}$, can also be approximately described by Eq. (14) replacing the proton saturation scale by a nucleus one:

$$
Q_{s, p}^{2} \rightarrow Q_{s, A}^{2}(x)=A^{1 / 3} c(b) Q_{s, p}^{2}(x),
$$

where $c=c(b)$ is the profile function of impact parameter $b$ (for central collisions, we use $c=0.85$ following Ref. [38]). Moreover, in practical calculations we adopt the CT10 NLO parametrization for the parton distributions and the KniehlKramer-Potter (KKP) fragmentation function $D_{h / f}\left(z_{h}, \mu_{F}^{2}\right)$ of a quark into a neutral pion [56]. In our analysis, the minimal transverse momentum ( $p_{T}^{\text {cut }}$ ) for the photon and the pion in Eq. (8) will be assumed to be the same and equal to 1.0 (3.0) GeV for RHIC (LHC) energies.

In Fig. 6 we present our predictions using the GBW model for the correlation function in the case of $p p$ and $p A u$ collisions at RHIC $(\sqrt{s}=0.2 \mathrm{TeV})$ and for two configurations for the photon and pion rapidities. We consider two distinct kinematical configurations, first, when both photon and pion are produced at forward rapidities, with $\eta=y_{\pi}=3$, and, second, when the photon is produced at forward rapidity $(\eta=3)$ but the pion is produced at central rapidity $\left(y_{\pi}=0\right)$. Such configurations can be experimentally studied by the STAR Collaboration in both $p p$ and $p A$ collisions. It is important to emphasize that the saturation scale increases for smaller values of $x_{g}$, with $x_{g}=x_{1} e^{-2 \eta}+\frac{x_{h}}{z_{h}} e^{-2 y_{h}}$, and for larger nuclei. Therefore, larger pion and photon rapidities imply the increasing saturation scale. Consequently, one should expect a larger decorrelation at forward rapidities and at larger values of the atomic mass $A$.

In addition, for forward rapidities, the transverse momentum of the produced particles is limited by the phase space and, in general, does not assume a large value. Therefore, for this kinematical range, the saturation scale becomes non-negligible in comparison to the typical transverse momentum of the back-to-back scattered particles. In this case, the saturation scale induces a noticeable decorrelation between them. Such an expectation is confirmed by

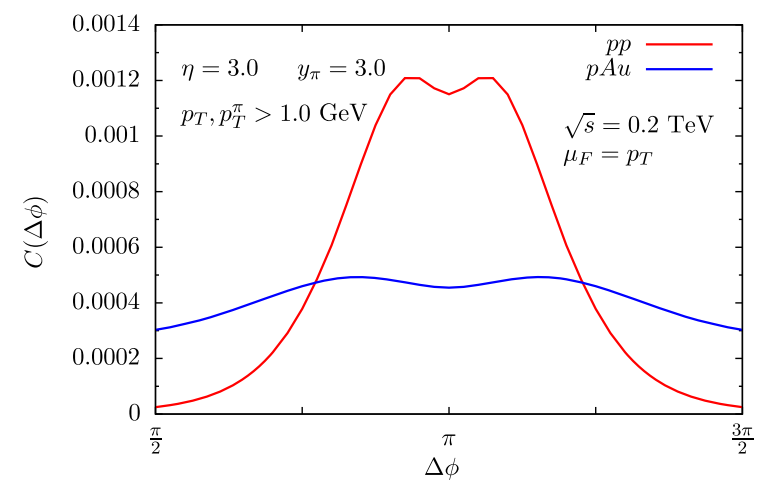

FIG. 6. The correlation function $C(\Delta \phi)$ for the associated isolated photon and pion production in $p p$ and $p A u$ collisions at RHIC $(\sqrt{s}=0.2 \mathrm{TeV})$ considering two different configurations for the photon and pion rapidities. 

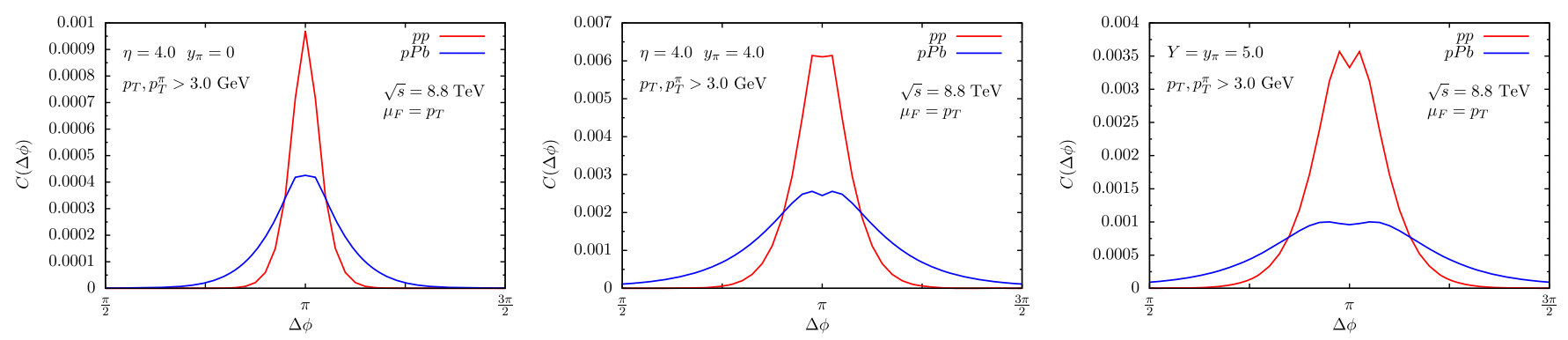

FIG. 7. The correlation function $C(\Delta \phi)$ for the associated photon and pion production in $p p$ and $p P b$ collisions at the LHC $(\sqrt{s}=8.8 \mathrm{TeV})$ considering three distinct configurations for the isolated photon and pion rapidities.

the results presented in Fig. 6. For the two configurations of rapidities mentioned above, we predict the presence of a double peak in the correlation function in $p p$ collisions with a dip at $\Delta \phi=\pi$, in consistency with the DY + pion analysis of Refs. [22,24]. Moreover, the width of the double peak increases when both rapidities are large. For $p A u$ collisions, the decorrelation grows, with the correlation function being almost flat for $\eta=y_{\pi}=3$. Such a large

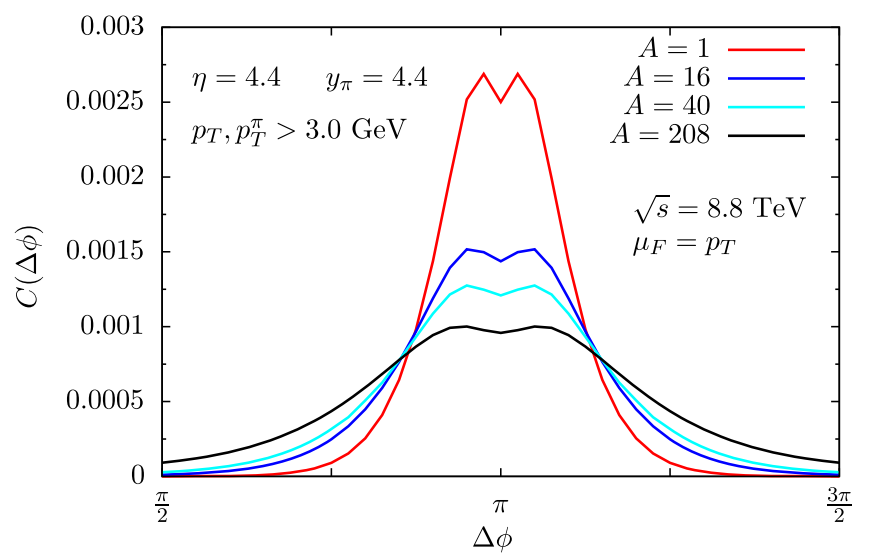

FIG. 8. The correlation function $C(\Delta \phi)$ for the associated photon and pion production in $p A$ collisions at the LHC $(\sqrt{s}=8.8 \mathrm{TeV})$ for different nuclei. decorrelation can, in principle, be probed in future experimental measurements at RHIC.

Our predictions for the correlation function in $p p$ and $p P b$ collisions at $\sqrt{s}=8.8 \mathrm{TeV}$ are presented in Fig. 7. In the case of $p p$ collisions, we notice a smearing of the backto-back correlation when the rapidities are increased, which is directly related to the growth of the saturation scale. A similar behavior is predicted for the DY + pion process $[22,24]$. In contrast, for $p P b$ collisions at the same centerof-mass energy, we predict a larger decorrelation, in particular for $\eta=y_{\pi}=5$.

To analyze the impact of the atomic mass on the correlation function, in Fig. 8 we present our predictions for $C(\Delta \phi)$ in $p A$ collisions at $\sqrt{s}=8.8 \mathrm{TeV}$, different values of $A$, and $\eta=y_{\pi}=4.4$. As expected due to a growth of the saturation scale with $A$, we observe that the decorrelation becomes stronger for heavier nuclei. Such a result indicates that, in principle, the study of $C(\Delta \phi)$ for a fixed energy and for a given set of rapidities can be used to probe the $A$ dependence of the nuclear saturation scale $Q_{s, A}$.

Finally, let us discuss how the above predictions for the correlation function depend on the modeling of the UGDF in the target. For the latter, so far we have used Eq. (14) inspired by the GBW model as input in our calculations. It is interesting to compare these results with those obtained by using the solution of the rcBK equation discussed above. In Fig. 9 we present a comparison between the GBW predictions and those derived using the AAMQS model.
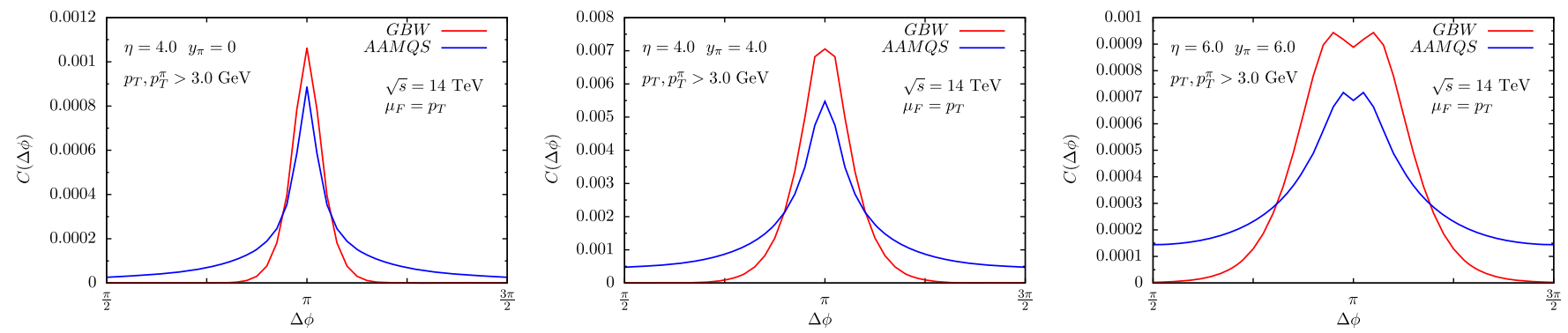

FIG. 9. The correlation function $C(\Delta \phi)$ for the associated photon and pion production in $p p$ collisions at the $\mathrm{LHC}(\sqrt{s}=14 \mathrm{TeV})$ considering three configurations for the photon and pion rapidities and the GBW and AAMQS models for the UGDF in the proton target. 
We observe that both models predict a similar behavior for the correlation function and differ mainly in the near-side $(\Delta \phi=0)$ region, which is dominated by the leading jet fragmentation. Such a result is anticipated from the previous studies $[38,40,41]$, which have demonstrated that the behavior of $C(\Delta \phi)$ in the away-side $(\Delta \phi=\pi)$ region is not strongly dependent on the large transverse momentum tail of the UGDF.

Recently, the formalism of resummation of the Sudakovtype double logarithms at small- $x$ has been developed $[57,58]$. Such terms appear in the description of the transverse-momentum spectrum of a given hard process due to the presence of two scales. In the considered process, these two scales are the total transverse-momentum imbalance of the $\gamma+\pi$ system, $q_{T}=\left|\vec{p}_{T}+\vec{p}_{T}^{\pi}\right|$, and the average transverse momentum $Q_{T}=\left|\vec{p}_{T}-\vec{p}_{T}^{\pi}\right| / 2$. In the kinematical domain of a large scale separation, $Q_{T} \gg q_{T}$, large logarithms of type $\ln ^{2} Q_{T}^{2} / q_{T}^{2}$ appear in every order of perturbative calculations and need to be resummed. As demonstrated in Ref. [58] for jet-photon production in $p A$ collisions when considering the corrections associated with single gluon radiation, the contribution of the Sudakov double logarithms can be factorized from the small- $x$ logarithms provided that these two contributions are well separated in the phase space of the radiated gluon. This formalism was used in Ref. [40] to estimate the di-hadron angular correlations in forward $p A$ collisions, which demonstrated that the Sudakov correction becomes important in some specific regions of the phase space. One important question is the impact of the Sudakov corrections on the dip structure observed in our results. As demonstrated above, the dip appears when the typical transverse momenta of the photon and pion are relatively close to the saturation scale. Moreover, we have noticed that the total transverse momentum imbalance $q_{T}$ is of the order of $Q_{s}$ indicating that the dip arises when $Q_{T} \approx q_{T}$. In this regime, the Sudakov logarithms $\ln ^{n} Q_{T}^{2} / q_{T}^{2}$ are typically small. Consequently, we do not expect any significant effect on the angular distributions and, in particular, on the dip structure associated with the Sudakov resummation in the relevant kinematic domains. Surely, the inclusion of such small corrections can be considered for a future work, with the results presented here being the starting point.

\section{SUMMARY}

In this paper, we performed a detailed phenomenological analysis of the isolated photon production in $p p$ and $p A$ collisions at typical RHIC and LHC energies in the framework of the color dipole approach. We employed three different phenomenological saturation models for the dipole-target scattering and analyzed differential distributions of prompt photons in transverse momentum $p_{T}$. Besides, we have investigated the correlation function $C(\Delta \phi)$ in the azimuthal angle between the real high- $p_{T}$ photon produced in association with a leading pion emerging via fragmentation of a projectile quark which emits the photon. This observable has been studied in $p p$ and $p A$ collisions at RHIC and LHC energies and at different rapidities of final states. In $p p$ collisions, the correlation function exhibits a double-peak structure close to $\Delta \phi \simeq \pi$ in certain kinematical configurations corresponding to both the real high- $p_{T}$ photon and the accompanied high- $p_{T}$ pion being produced at forward rapidities. In the case of $p A$ collisions, a larger nuclear saturation scale enforces a stronger decorrelation between the photon and the pion. The correlation function is a more exclusive observable than the standard transverse momentum spectra of the isolated photon and appears to be strongly sensitive to the details of theoretical modeling of the saturation phenomena in QCD. A future measurement of this observable at different RHIC and LHC energies would be capable of setting stronger constraints on the unintegrated gluon density in the small- $x$ and small- $k_{T}$ domains as well as on the dipole model parametrizations, thus, enabling one to directly probe the saturation scale.

\section{ACKNOWLEDGMENTS}

V.P. G. thanks the members of the Faculty of Nuclear Sciences and Physical Engineering of the Czech Technical University in Prague and of the Nuclear Physics Institute of the CAS for warm hospitality during the completion of this work. V. P. G. and Y. L. were partially financed by the Brazilian funding agencies CNPq, CAPES, FAPERGS, and INCT-FNA (Process No. 464898/2014-5). M. Š. is partially supported by Grants No. LTT17018 and No. LTT18002 of the Ministry of Education of the Czech Republic and by Grant No. 13-20841S of the Czech Science Foundation (GACR). R. P. is partially supported by the Swedish Research Council Grant No. 2016-05996, by the European Research Council (ERC) under the European Union's Horizon 2020 research and innovation programme (Grant No. 668679), as well as by the Ministry of Education, Youth and Sports of the Czech Republic Project No. LTT17018 and by the NKFI Grant No. K133046 (Hungary). Part of this work has been performed in the framework of COST Action CA15213 "Theory of hot matter and relativistic heavy-ion collisions" (THOR). 
[1] R. Pasechnik and M. Šumbera, Universe 3, 7 (2017).

[2] S. Acharya et al. (ALICE Collaboration), Phys. Rev. C 99, 024912 (2019).

[3] G. David, Rep. Prog. Phys. 83, 046301 (2020).

[4] E. L. Berger and J. w. Qiu, Phys. Lett. B 248, 371 (1990).

[5] L. E. Gordon and W. Vogelsang, Phys. Rev. D 50, 1901 (1994).

[6] S. Frixione, Phys. Lett. B 429, 369 (1998).

[7] F. Gelis and J. Jalilian-Marian, Phys. Rev. D 66, 014021 (2002).

[8] B. Ducloue, T. Lappi, and H. Mantysaari, Phys. Rev. D 97, 054023 (2018).

[9] J. C. Peng and J. W. Qiu, Prog. Part. Nucl. Phys. 76, 43 (2014).

[10] S. Benić, K. Fukushima, O. Garcia-Montero, and R. Venugopalan, Phys. Lett. B 791, 11 (2019).

[11] C. Schmidt, J. Pumplin, D. Stump, and C. P. Yuan, Phys. Rev. D 93, 114015 (2016).

[12] D. d'Enterria and J. Rojo, Nucl. Phys. B860, 311 (2012).

[13] G. Aad et al. (ATLAS Collaboration), Phys. Rev. D 83, 052005 (2011).

[14] V. Khachatryan et al. (CMS Collaboration), Phys. Rev. Lett. 106, 082001 (2011).

[15] J. Adam et al. (ALICE Collaboration), Phys. Lett. B 754, 235 (2016).

[16] C. Zhang (ALICE FoCal Group), Nucl. Instrum. Methods A 845, 542 (2017).

[17] S. S. Adler et al. (PHENIX Collaboration), Phys. Rev. Lett. 98, 012002 (2007).

[18] A. Adare et al. (PHENIX Collaboration), Phys. Rev. D 82, 072001 (2010).

[19] A. Adare et al. (PHENIX Collaboration), Phys. Rev. Lett. 123, 022301 (2019).

[20] Q. Yang (STAR Collaboration), Nucl. Phys. A982, 951 (2019).

[21] G. Roland (Sphenix Collaboration), Proc. Sci., HardProbes2018 (2019) 013.

[22] E. Basso, V. P. Goncalves, M. Krelina, J. Nemchik, and R. Pasechnik, Phys. Rev. D 93, 094027 (2016).

[23] V. P. Goncalves, M. Krelina, J. Nemchik, and R. Pasechnik, Phys. Rev. D 94, 114009 (2016).

[24] E. Basso, V. P. Goncalves, J. Nemchik, R. Pasechnik, and M. Sumbera, Phys. Rev. D 93, 034023 (2016).

[25] C. A. Salgado et al., J. Phys. G 39, 015010 (2012).

[26] A. B. Zamolodchikov, B. Z. Kopeliovich, and L. I. Lapidus, Sov. Phys. JETP Lett. 33, 595 (1981).

[27] S. J. Brodsky, A. Hebecker, and E. Quack, Phys. Rev. D 55, 2584 (1997).

[28] B. Z. Kopeliovich, A. Schafer, and A. V. Tarasov, Phys. Rev. C 59, 1609 (1999).

[29] B. Z. Kopeliovich, J. Raufeisen, and A. V. Tarasov, Phys. Lett. B 503, 91 (2001).

[30] B. Z. Kopeliovich, J. Raufeisen, A. V. Tarasov, and M. B. Johnson, Phys. Rev. C 67, 014903 (2003).

[31] J. Raufeisen, J.-C. Peng, and G. C. Nayak, Phys. Rev. D 66, 034024 (2002); M. B. Johnson, B. Z. Kopeliovich, M. J. Leitch, P. L. McGaughey, J. M. Moss, I. K. Potashnikova, and I. Schmidt, Phys. Rev. C 75, 035206 (2007); M. B.
Johnson, B. Z. Kopeliovich, and I. Schmidt, Phys. Rev. C 75, 064905 (2007).

[32] N. N. Nikolaev and B. G. Zakharov, Z. Phys. C 64, 631 (1994).

[33] K. J. Golec-Biernat and M. Wusthoff, Phys. Rev. D 59, 014017 (1998).

[34] N. N. Nikolaev and B. G. Zakharov, Zh. Eksp. Teor. Fiz. 105, 1117 (1994) [J. Exp. Theor. Phys. 78, 598 (1994)]; Z. Phys. C 64, 631 (1994).

[35] N. N. Nikolaev, G. Piller, and B. G. Zakharov, Z. Phys. A 354, 99 (1996); B. Z. Kopeliovich and A. V. Tarasov, Nucl. Phys. A710, 180 (2002).

[36] R. S. Pasechnik and B. Z. Kopeliovich, Eur. Phys. J. C 71, 1827 (2011); R. S. Pasechnik, B. Z. Kopeliovich, and I. K. Potashnikova, Phys. Rev. D 86, 114039 (2012); R. Pasechnik, B. Z. Kopeliovich, and I. K. Potashnikova, Phys. Rev. D 92, 094014 (2015); R. Pasechnik, B. Kopeliovich, and I. Potashnikova, Adv. High Energy Phys. 2015, 1 (2015); B. Z. Kopeliovich, R. Pasechnik, and I. K. Potashnikova, Phys. Rev. D 98, 114021 (2018).

[37] C. Marquet, Nucl. Phys. A796, 41 (2007); J. L. Albacete and C. Marquet, Phys. Rev. Lett. 105, 162301 (2010).

[38] A. Stasto, B. W. Xiao, and F. Yuan, Phys. Lett. B 716, 430 (2012).

[39] A. Stasto, B-W Xiao, and D. Zaslavsky, Phys. Rev. D 86, 014009 (2012).

[40] A. Stasto, S. Y. Wei, B. W. Xiao, and F. Yuan, Phys. Lett. B 784, 301 (2018).

[41] J. Jalilian-Marian and A. H. Rezaeian, Phys. Rev. D 86, 034016 (2012); A. H. Rezaeian, Phys. Rev. D 86, 094016 (2012).

[42] F. Gelis, E. Iancu, J. Jalilian-Marian, and R. Venugopalan, Annu. Rev. Nucl. Part. Sci. 60, 463 (2010); E. Iancu and R. Venugopalan, Quark Gluon Plasma, edited by R. C. Hwa et al. (World Scientific, Singapore, 2004), pp. 249-3363; H. Weigert, Prog. Part. Nucl. Phys. 55, 461 (2005); J. JalilianMarian and Y. V. Kovchegov, Prog. Part. Nucl. Phys. 56, 104 (2006).

[43] J. L. Albacete, N. Armesto, J. G. Milhano, P. Quiroga-Arias, and C. A. Salgado, Eur. Phys. J. C 71, 1705 (2011).

[44] F. Gelis and J. Jalilian-Marian, Phys. Rev. D 66, 094014 (2002); J. Jalilian-Marian, Nucl. Phys. A739, 319 (2004); F. Gelis and J. Jalilian-Marian, Phys. Rev. D 76, 074015 (2007).

[45] F. Dominguez, J.-W. Qiu, B.-W. Xiao, and F. Yuan, Phys. Rev. D 85, 045003 (2012).

[46] D. Zaslavsky, arXiv:1409.8259.

[47] I. I. Balitsky, Phys. Rev. Lett. 81, 2024 (1998); Phys. Lett. B 518, 235 (2001); I. I. Balitsky and A. V. Belitsky, Nucl. Phys. B629, 290 (2002).

[48] Y. V. Kovchegov, Phys. Rev. D 60, 034008 (1999); 61, 074018 (2000).

[49] E. Iancu, K. Itakura, and S. Munier, Phys. Lett. B 590, 199 (2004).

[50] R. J. Glauber and G. Matthiae, Nucl. Phys. B21, 135 (1970).

[51] A. H. Mueller, Nucl. Phys. B335, 115 (1990).

[52] K. Dusling, F. Gelis, T. Lappi, and R. Venugopalan, Nucl. Phys. A836, 159 (2010). 
[53] T. Lappi and H. Mantysaari, Phys. Rev. D 88, 114020 (2013).

[54] H. L. Lai, M. Guzzi, J. Huston, Z. Li, P. M. Nadolsky, J. Pumplin, and C.-P. Yuan, Phys. Rev. D 82, 074024 (2010).

[55] S. S. Adler et al. (PHENIX Collaboration), Phys. Rev. Lett. 98, 012002 (2007); A. Adare et al. (PHENIX Collaboration), Phys. Rev. D 86, 072008 (2012).
[56] B. A. Kniehl, G. Kramer, and B. Potter, Nucl. Phys. B582, 514 (2000).

[57] A. Mueller, B. Xiao, and F. Yuan, Phys. Rev. Lett. 110, 082301 (2013).

[58] A. Mueller, B. Xiao, and F. Yuan, Phys. Rev. D 88, 114010 (2013). 